\title{
Survey on Massive MIMO: Technology, Challenges, Opportunities and Benefits
}

\author{
Karthik Kumar Vaigandla ${ }^{1 *}$, Dr.N.Venu ${ }^{2}$ \\ ${ }^{1,2}$ Dept. of Electronics \& Communication Engineering, Balaji Institute of Technology \\ and Science, Warangal, Telangana, India \\ ${ }^{1}$ vkvaigandla@bitswgl.ac.in, ${ }^{2}$ venunookala@gmail.com
}

\begin{abstract}
Wireless communication technologies have been studied and explored in response to the global shortage of bandwidth in the field of wireless access. Next-generation networks will be enabled by massive MIMO. Using relatively simple processing, it provides high spectral and energy efficiency by combining antennas at the receiver and transmitter. This paper discusses enabling technologies, benefits, and opportunities associated with massive MIMO, and all the fundamental challenges. Global enterprises, research institutions, and universities have focused on researching the $5 G$ mobile communication network. Massive MIMO technologies will utilize simpler and linear algorithms for beam forming and decoding. As part of future $5 G$, massive MIMO technology will be used to increase the efficiency of spectrum utilization and channel capacity. The paper then summarizes the technologies that are used in massive MIMO system, including channel estimation, pre-coding, and signal detection.
\end{abstract}

Keywords: Beam forming; channel estimation; massive MIMO; pilot contamination; precoding; signal detection; spectral efficiency;5G;opportunities;challenges.

\section{INTRODUCTION}

Increasing mobile usage and the size of related industries can be traced to rapid developments in mobile communication technology. Due to this, wireless communication systems have to handle higher transmission rates and greater capacities as well as make better use of bandwidth resources [1]. The system must improve its spectrum utilization due to the shortage of spectrum resources. It is possible to meet users' performance demands with traditional MIMO technology using limited spectrum resources. A traditional MIMO system, however, has fewer antennas, resulting in a lower performance level. Massive MIMO is a key technology that will be utilized in future $5 \mathrm{G}$ networks for its ability to satisfy the requirements of future wireless business models, maximize spectrum efficiency and enhance system capacity, and enhance link and data transmission reliability [2].

A popular technique for telecommunications in the modern age is massive MIMO, which is applied in long-term evolution (LTE) and wireless local area networks (WLAN). 5G communication relies heavily on this technology [41]. In order to meet the needs of 5G and beyond, massive MIMO is the most enthralling wireless access technology. MIMO technology is being extended with massive MIMO, an innovative method of enhancing spectral efficiency and throughput by using hundreds and sometimes thousands of antennas. The technology involves combining antennas, radios, and spectrum in order to create a system that provides higher speeds and capacities for the upcoming 5G [3],[4] . In order for wireless standards to be standardized, massive MIMO is essential due to its capacity 
increases throughput and spectral efficiency [5],[6]. Massive MIMO with multiple antennas achieves considerable array gains [7]. Security purposes make use of massive MIMO to identify the legitimate user with a beam guide that is as accurate as possible. This significantly reduces the probability of unauthorized access to information (such as Eve). Comparing massive MIMO to traditional MIMO, there are several significant challenges: (1) it is hard to determine the CSI process; (2) antenna distances are too short to exclude channel modeling from evaluation. The current state of massive MIMO attacks uses both active and passive eavesdropping techniques [8]. With MIMO technology, wireless communication range and speeds are greatly enhanced, and additional bandwidth and power isn't necessary. 5G's complex system relies heavily on the massive MIMO scheme. High spectral efficiency can be achieved by transmitting different signals simultaneously over multiple transmit antennas and receiving them at multiple receive antennas simultaneously. MIMO systems need to be highly effective in terms of spectral efficiency [40].

The 5G networks will not be able to provide a completely automated, everything-as-aservice, and genuinely immersive service [42]. Despite the fact that the 5G communication system will have numerous advantages over present systems, it will not be able to meet the needs of future intelligent and automated concepts in ten years [42]. In comparison to 4G communications, the 5G network will deliver additional capabilities and higher service quality [42]. Additional frequency bands, new spectrum management strategies, and the combination of licensed and unlicensed channels are all new features of 5G technology [42].

The spectral efficiency of massive MIMO is over 10 times higher than that of pointto-point MIMO in realistic propagation environments with simpler signal processing algorithms [9]-[12]. This paper will summarize Massive MIMO's progress, along with the interesting opportunities and challenges it faces, as one of the key technologies in $5 \mathrm{G}$ wireless communication. The American multinational tech giant Cisco has predicted that by 2025 more people will own smart phones than electricity. Also, 75\% of mobile data traffic will be video data which can consume a great deal of bandwidth. Customers will expect wireless services to be of wire line quality, and more reliable connections and higher bit rates will be expected. Massive MIMO systems, which provide tens of gigabits of data per second without consuming much additional spectrum, have the potential to deliver these bit rates in real-time wireless multimedia services [13].

\section{MASSIVE MIMO TECHNOLOGY}

A Bell Labs scientist called Marzetta proposed the concept of massive MIMO in 2010 [14]. A limited number of antennas per cell has therefore been found to have some distinct features. An impressive number of antennas is a characteristic of the massive MIMO technology [15]. This represents an order of magnitude higher number of antennas than the number in the existing system. Generally, mobile terminals use single antenna communication to serve multiple users at once using the same time-frequency resource.

With its flexibility and ability to enable new games and structures, massive MIMO has potential for 5G. As devices become more advanced, the center level as well as higher level plans should reflect this. 5G structures for D2D and telecommunications are proposed [39]. The availability of M2M (machine-to-machine) communication services should also be an important aspect of $5 \mathrm{G}$.

As with massive MIMO, large scale MIMO is a promising way to boost spectral efficiency. A large number of antennas will be added to the base station. A large-scale statistical effect is invoked using this technology. The transmission is mostly free of fading, interference, and noise, and transmitted energy is focused only on the intended target. The research community has been paying much attention to massive MIMO, and many are 
examining its potential. The basic premise has been made more practical in many ways. On the problem of the estimation of CSI characteristics of the many channels, progress has been made. An examination of energy efficiency aspects is being conducted in addition to the problem of computing cost for precoding schemes [16].

Uplink Transmission: Users communicate with the base station via the uplink channel, which sends data and pilot signals. Consider a base station equipped with $\mathrm{M}$ antennas and communicating with the $\mathrm{N}$ users using single antennas at the same time. A base station receives the following signal during uplink if the user or pilot signals are $\mathrm{x}$.

$$
\begin{aligned}
& y=H . x+n_{\text {uplink }} \\
& n_{\text {uplink }}=n_{\text {uplink-interference }}+n_{\text {noise }}
\end{aligned}
$$

Where $\mathrm{y}$ is the user terminal receives a signal, $\mathrm{H}$ is the channel vector between the base station and the user terminal; and $\mathrm{n}_{\text {uplinkis }}$ used for interference produced by several transmissions and receiver noise, describes the additional interference. Interference added based on individual signals $x$ can be independent of channel $\mathrm{H}$.

Downlink Transmission: Data is transmitted or estimated between a user and a base station via the downlink channel. The base station estimates the channel through training pilots. The UE transmits data to the base station downlink. A massive MIMO system with a downlink; base station is having $\mathrm{M}$ antennas and user having $\mathrm{N}$ antennas. Multiple users simultaneously receive independent information from the base station. The received signal is

$$
\begin{aligned}
& y_{k}=h_{k} \cdot x_{k}+n_{\text {downlink }} \\
& n_{\text {downlink }}=n_{\text {downlink-interference }}+n_{\text {noise }}
\end{aligned}
$$

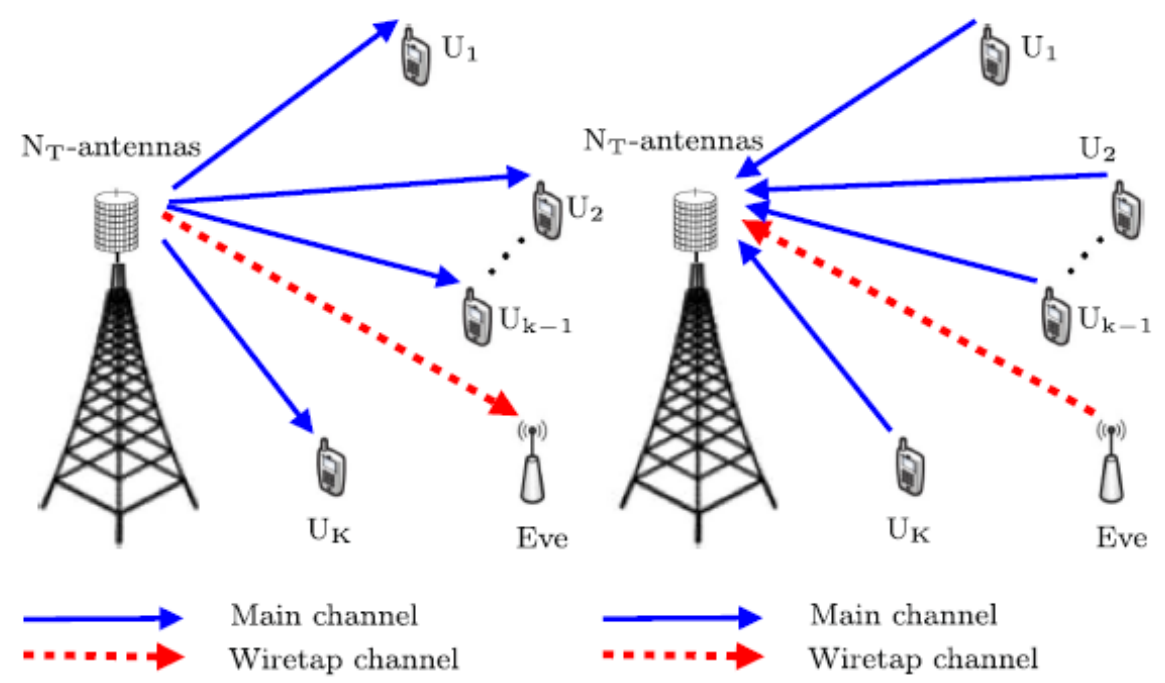

a) Downlink phase b) Uplink phase

Figure 1. Massive MIMO downlink and uplink 


\section{OPPORTUNITIES AND BENEFITS OF MASSIVE MIMO}

\subsection{Opportunities of Massive MIMO}

Increase in system capacity, spectral efficiency and energy efficiency: Wireless MIMO systems use many antennas with numbers orders of magnitude larger than those used by cellular base stations [11]. Both uplink and downlink communications can be improved with it by increasing spectral and energy efficiency [12]. Massively multi-user MIMO-based systems greatly increase the system capacity by utilizing highly multiplexed spatial signals. The linear beamforming and precoding capabilities of the BSs were demonstrated using a large array of antennas [9], [11], [12]. Many simulation studies have focused on the spectral efficiency of large antenna arrays. Two different precoding schemes, eigen beamforming (BF) and zero forcing (ZF) were used to simulate a uniform linear array (ULA) at $2.6 \mathrm{GHz}$, serving 10 user terminals. The $\mathrm{ZF}$ and $\mathrm{BF}$ were able to achieve spectra of $58 \mathrm{~b} / \mathrm{s} / \mathrm{Hz}$ and 48 $\mathrm{b} / \mathrm{s} / \mathrm{Hz}$, respectively. In comparison with a single antenna, the multi-antenna BS offers a greater spectral capacity. As the number of customers simultaneously served by BS antennas increases, the spectral capacity doubles almost linearly. ZF precoding generally performs better than MRC or BF precoding with many antennas.

Cost effective and energy efficient (low power consumption) components: Using power efficient and cost-effective components, a massive MIMO system can be built [12]. MIMO systems with very high throughput are typically composed of antenna arrays consisting of individual antenna elements with infrequent power use, typically in the milli watt range. Reducing transmit power is necessary to improve the quality of service (QoS) of the system due to the increased energy consumption of cell towers [17]. MIMO system is quite complex at one end and consumes a lot of energy at the other end; however, massive MIMO reduces the complexity of both ends.

\subsection{Benefits of Massive MIMO}

Spectral Efficiency: By focusing narrow beams of light toward a user, massive MIMO provides higher spectral efficiency.

Energy Efficiency: Massive MIMO systems reduce energy consumption by focusing antenna array onto a small section, thus requiring less energy radiated.

High Data Rate: Massive MIMO provides greater gain and multiplexing capacity to wireless systems, resulting in higher data rates and greater capacity.

User Tracking: Massive MIMO makes tracking users more reliable and accurate by using narrow signal beams.

Low Power Consumption: In massive MIMO, linear amplifiers are used to reduce the need for

bulky electronic equipment. Therefore, there is a considerable reduction in power consumption.

Less Fading: Massive MIMO is robust against fading due to a large number of antenna at the receiver [18].

Low Latency: The latency on the air interface is reduced by massive MIMO [19].

Reliability: When MIMO uses multiple antennas simultaneously, it provides more diversity gain, increasing the reliability [20].

Enhanced Security: Physical security is enhanced by massive MIMO's orthogonal channels and narrow beams [21].

Low Complex Linear Processing: The simple signal detectors and precoders are more efficient when there is more number of antennas on the base station. 


\section{CHALLENGES IN MASSIVE MIMO}

There are still many challenges and issues that need to be resolved in order to make massive MIMO a reality.

Pilot Contamination: The user pilots of neighboring cells may not be orthogonal in a multicell massive MIMO system. It is estimated that there are fewer orthogonal pilots than users. Pilot contamination occurs as a result of non-orthogonal pilots. Directed inter-cellular interference is caused by pilot contamination which, unlike other sources of interference, increases with the number of BS antennas and deteriorates the performance of the system. This problem has been resolved through the use of various estimation, precoding, and participation methods. However, it is worth studying more intensively methods that are more efficient, have low complexity, and limit or completely eliminate BS cooperation [22].

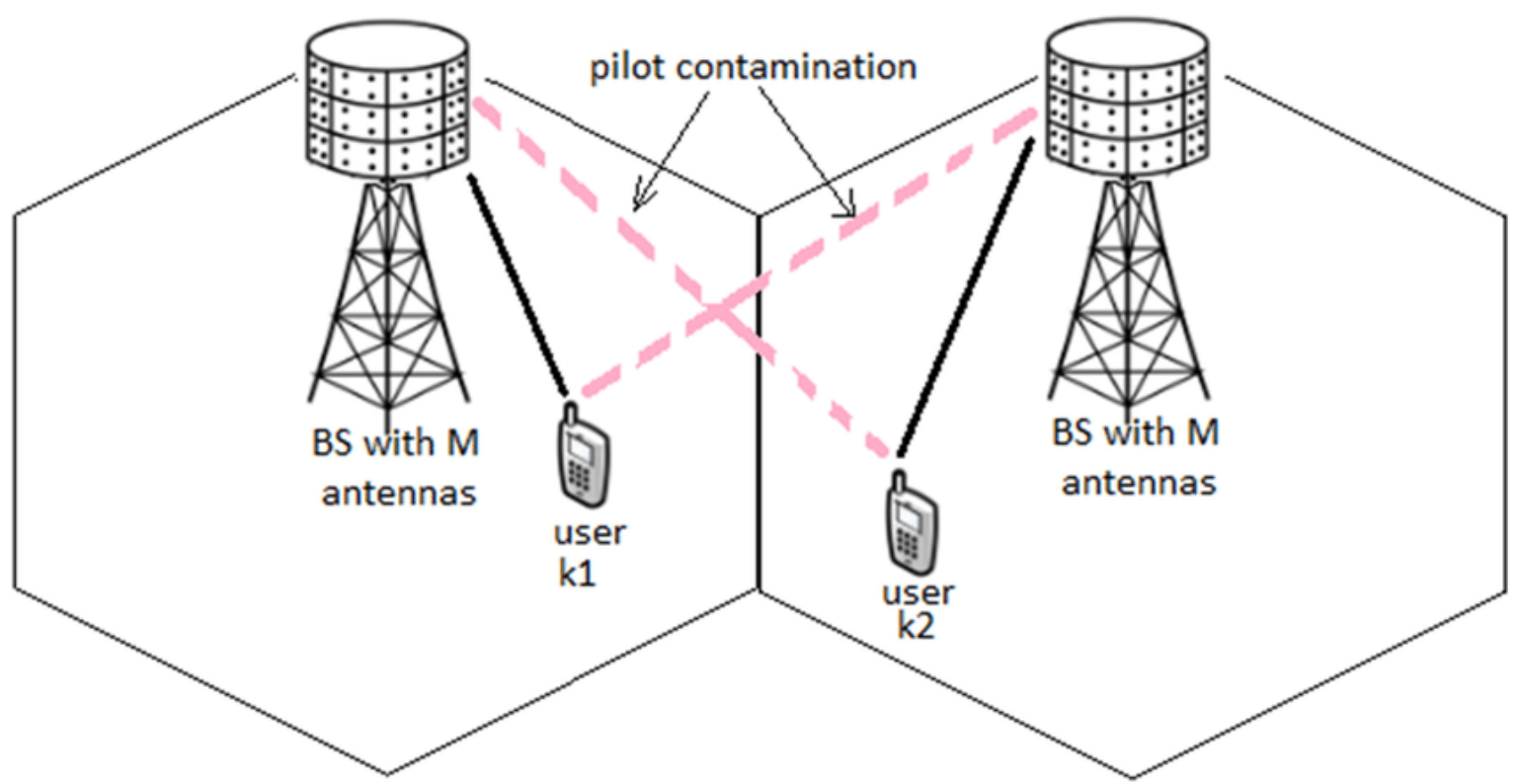

Figure 2. Massive MIMO pilot contamination

Channel Estimation: Channel State Information (CSI) is used in massive MIMO for signal detection and decoding. As the effect of fading, scattering, and other factors on the communication link is summed together, CSI is the information of the state of the communication link. A massive MIMO system will perform better if its CSI is perfect, and with a few more transmitting antennas or fewer receiving antennas, whichever is lower [23]. It is important to estimate CSI both during downlinks and uplinks of a frequency division duplexed system (FDD). With the help of orthogonal pilot signals transmitted by the user terminal, the base station decides what the channel to use during uplink. A pilot signal is sent by the base station to the user, during the downlink, and the user acknowledges the estimated channel information for the transmission. The downlink channel estimation strategy in FDD is very complex for massive MIMO systems with many antennas and cannot be applied in practice. MIMO systems were originally intended to use TDD operation, but research is underway to use FDD operations as well [24]. 
$T_{c}$

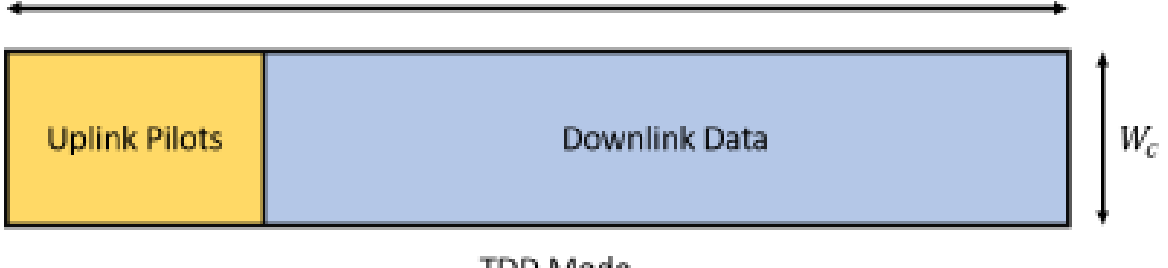

TDD Mode

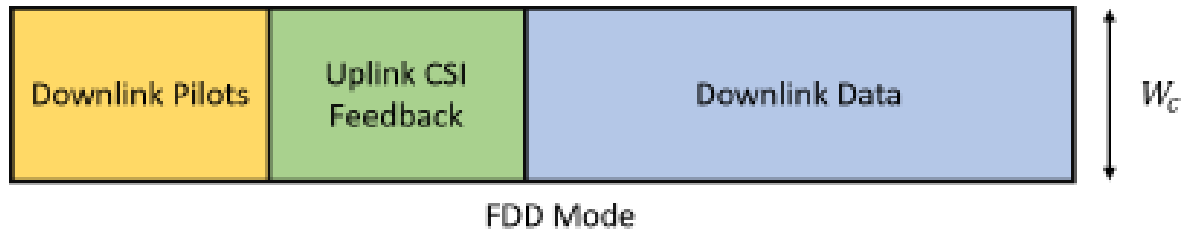

Figure 3. Typical pilot transmission and CSI feedback mechanism in FDD and TDD mode.
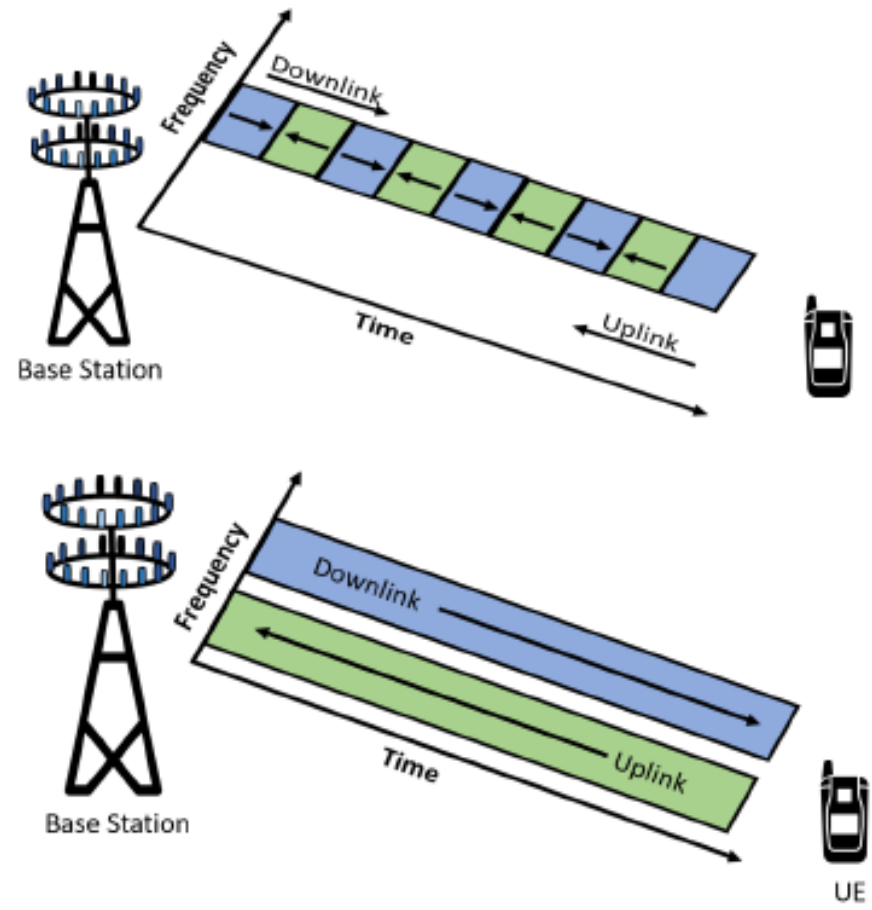

Figure 4. FDD \& TDD : Massive works best in TDD mode.

Precoding : Multi-antenna systems utilize precoding in order to support multi-stream transmission. As precoding mitigates path loss and interference in massive MIMO systems, and maximizes throughput, it has an important role to play. Based on the uplink pilot signals or feedback from the terminal, the base station estimates the CSI in MIMO systems. A few environmental factors affect the wireless channel management at the base station, which affects the received CSI at the base station [25]. The downlink performance of a base station is greatly influenced by the estimated CSI, even when the CSI is not perfect. It has been extensively studied how linear and non-linear precoding techniques can be used to mitigate the effects of pilot pollution on system performance when using massive MIMO precoding methods. 


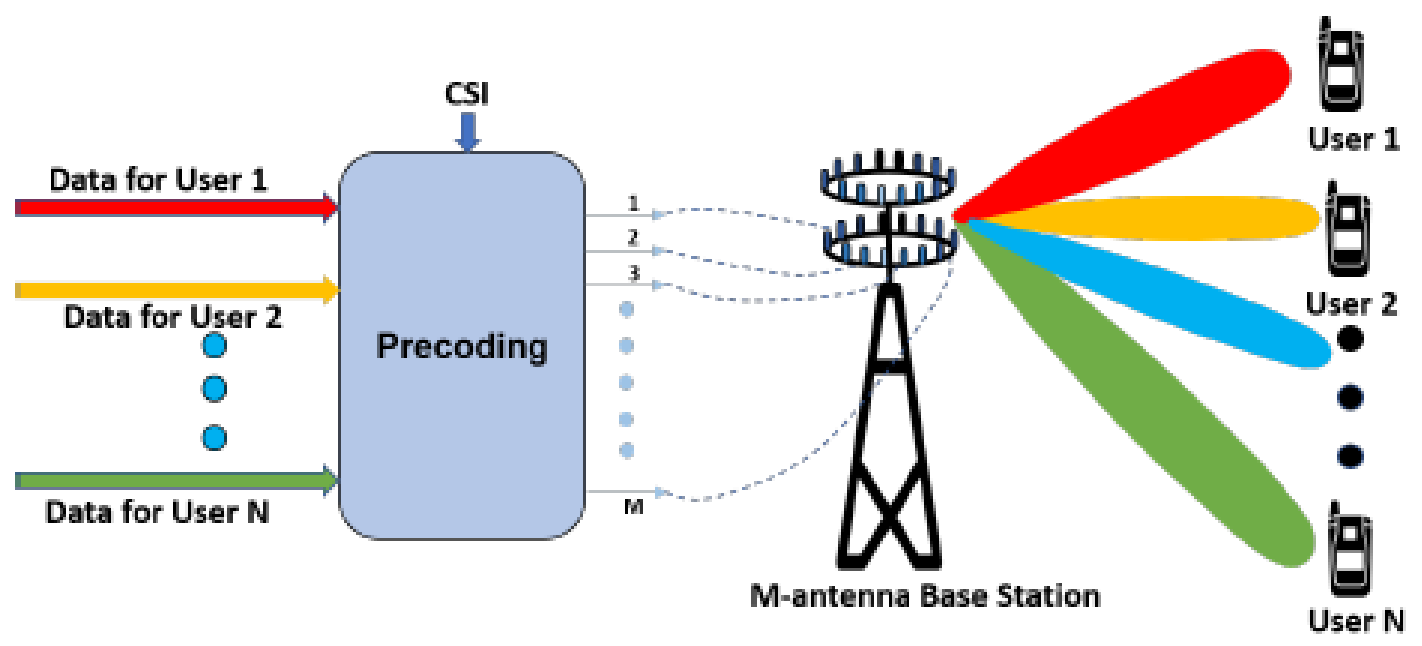

Figure 5. Precoding in a massive MIMO

User Scheduling : The base station of a massive MIMO network can communicate with multiple users simultaneously with many antennas. Multi-user communication simultaneously degrades the throughput performance and results in multi-user interference. Multi-user interference is reduced by using precoding during the downlink. Massive MIMO base stations have a limited number of antennas, so if the number of users exceeds the number of antennas, a proper user scheduling scheme must be implemented before precoding to increase throughput and sum rates.

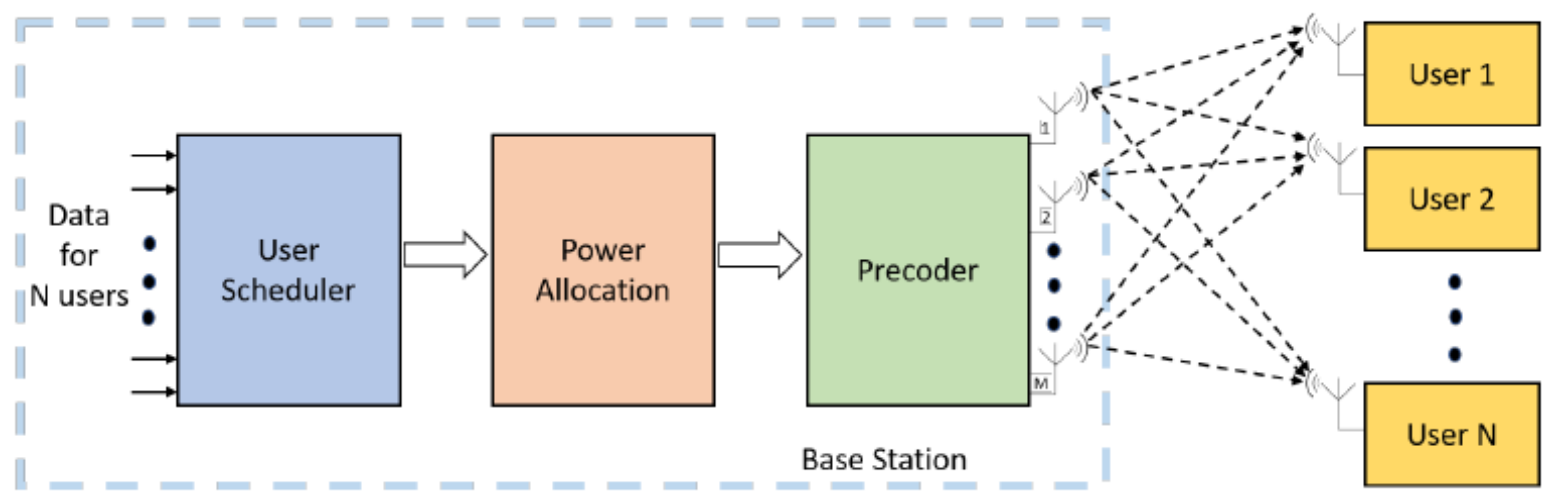

Figure 6. Massive MIMO user scheduling.

Hardware Impairments : Massive MIMO systems have received limited attention because of hardware impairments. This impairment affects the user more than the base station. There are actually more ways for the non-ideal case to mitigate the issue of pilot contamination than the ideal case [26]. A lot more research is needed on massive MIMO so it can become a reality. To reduce the effects of fading, noise, and interference, massive MIMO systems use a large number of antennas. Massive MIMO increases complexity and costs because of its large number of antennas. In order to reduce the computational complexity and the size of the hardware, massive MIMO technology should utilize small, low-cost components. When using an inexpensive component, poor quality would result [27]. Dedicated hardware, which can also run in parallel, is highly desirable to reduce the cost of the baseband signal processing. If the Peak to Average Power Ratio (PAPR) of a low-cost amplifier is low, one can reduce the impact of the amplifier on the transmitter. 


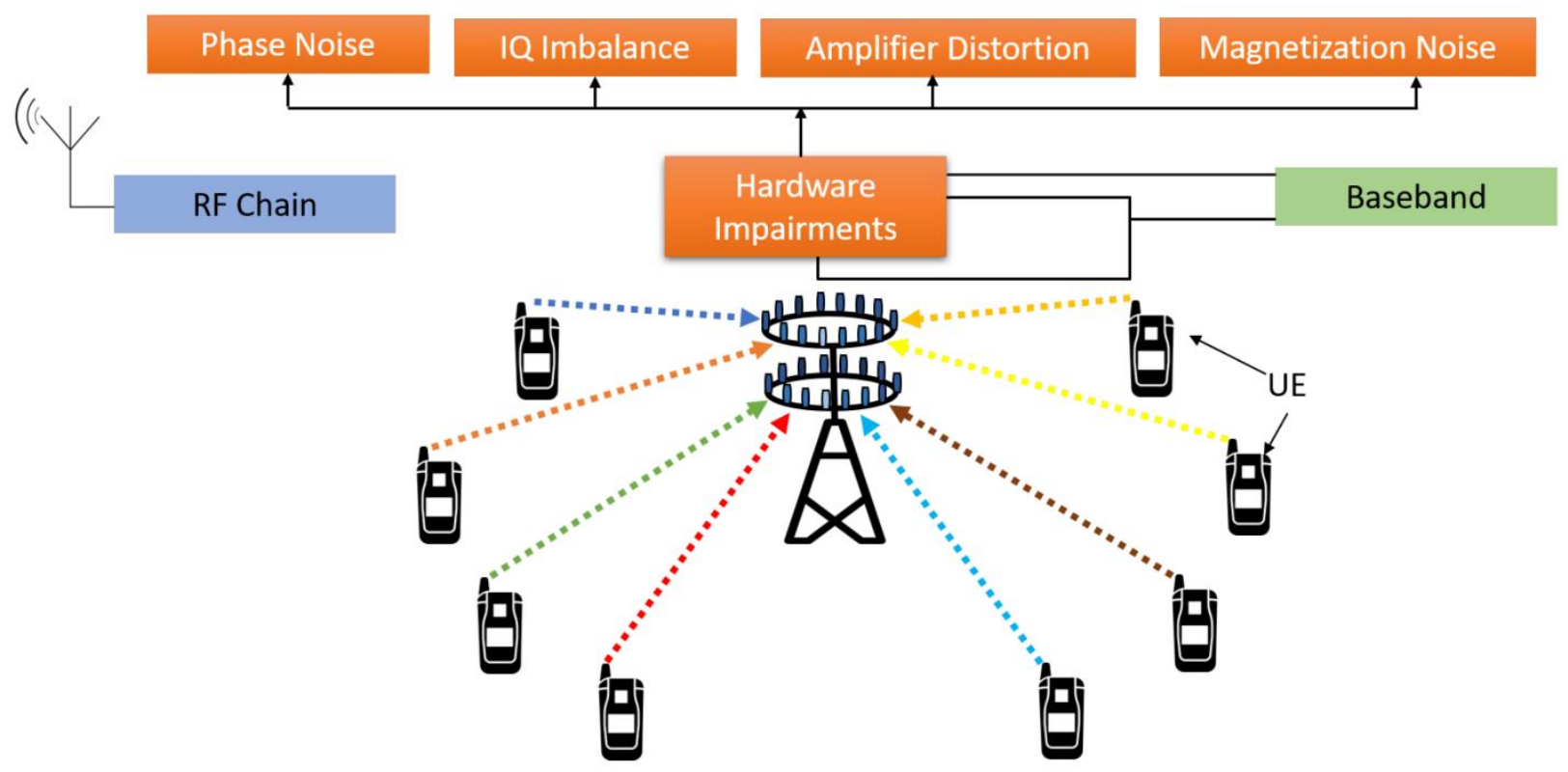

Figure 7. Massive MIMO hardware impairments

Modulation: It is necessary to use low-cost, power-efficient RF amplifiers for BSs equipped with a large number of antennas, since high PAPR may impede good performance for OFDM [28]. Despite low transmit power to noise power ratios, single carrier transmission achieves near optimal sum rate performance without requiring receiver equalization or multi-user resource allocation. The feasibility of such an optimization needs to be investigated further.

Energy Efficiency: Massive MIMO can be used to achieve greater spectral efficiency while consuming less power, resulting in significant energy savings. The spectral efficiency of a system always increases with an increasing number of antennas. In addition to an increase in antennas and users, the consumption of power increases. In the last few years, there have been several proposed methods to reduce the power consumption at the massive MIMO base station. These methods include precoding, detection, channel estimation, and user scheduling.

Signal Detection: Several different users are served by the base station and time-frequency resources are distributed. Massive MIMO is a system that gives the ability to detect received signals uplink by detecting the space signature of the transmitted signal from the cell terminal [1]. Massive MIMO systems are computationally complex due to more number of antennas, reducing the achievable throughput. Massive MIMO has considered several linear detectors, including ML, ZF, and MMSE. Massive MIMO detectors use ML and minimize error probability, but there are prohibitive costs for large antennas systems [29],[30]. Inter-antenna interference can be mitigated by ZF methods, but additive noise increases for ill-conditioned channel matrices [31]. Since it also takes into account noise power during the detection process [32], the MMSE detector has better performance than the ZF detector. 


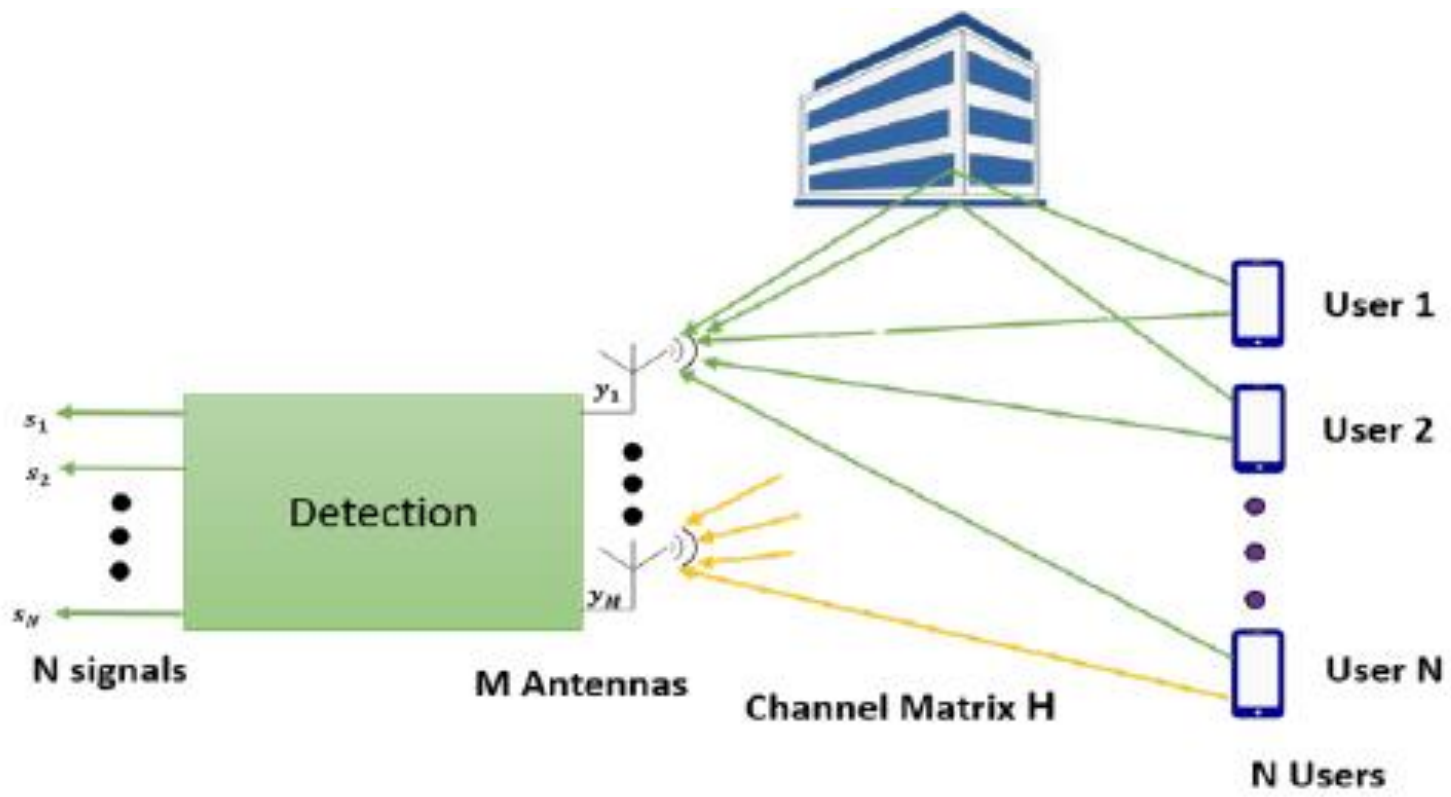

Figure 8. Signal detection in massive MIMO uplink

Antenna Arrays: Massive MIMO systems present a variety of practical problems related to antenna arrays. One is how the arrays are configured and deployed. It is suggested that antennas should be placed in a 2D grid for massive MIMO systems in [33]. Distributed arrays and 3D arrays can also be considered. In addition, mutual coupling between antenna elements needs to be addressed. Antennas should be well separated from each other in order to avoid the mutual coupling effect. Several antennas may be arranged together for massive MIMO systems, however, coupling must be considered [34]. As a final consideration, large antenna arrays have a considerable effect on system hardware and computing costs. It was proposed in [35],[36] that an electromagnetic lens antenna (ELA) could be used to reduce costs while still achieving high performance. Multipath separation and energy focusing functions can be performed by the proposed ELA. By reducing its implementation costs and complexity, it improves the performance of massive MIMO.

\section{CONCLUSION}

Future wireless communication will rely on massive MIMO technology. Wireless communication systems can be significantly improved in terms of channel capacity, energy efficiency, and spectrum utilization. This paper presents several different perspectives on massive MIMO systems. Wireless data traffic is likely to surge exponentially, creating a need for more capacity in the cellular spectrum. Large scale MIMO technology provides a solution for this growing global need for mobile data provision. The spectral and energy efficiency is achieved through the use of multiple antennas at both the transmitter and the receiver. Massive MIMO technology is currently less studied, despite the worldwide need for efficient spectrum. This emerging wireless access technology faces a number of open challenges. In this paper, overview of massive MIMO, enabling technologies, challenges and benefits are presented. 


\section{REFERENCES}

[1] Jiarui Wu, "Research on Massive MIMO Key Technology in 5G", IOP Conf. Series: Materials Science and Engineering, 2018, doi:10.1088/1757-899X/466/1/012083

[2] HUH H , CAIRE G, PAPADOPOULOSHC, etal. Achieving " massive mimo" spectral efficiency with a Not-so-LargeNumber of Antennas[ J]. IEEE Transactions on Wireless Communications , 2011, 11 (9); 3226 -3239

[3] A Closer Look at Massive MIMO. Available online: https://business.sprint.com/blog/massive-mimo (accessed on 20 January 2020).

[4] Rusek, F.; Persson, D.; Lau, B.K.; Larsson, E.G.; Marzetta, T.L.; Edfors, O.; Tufvesson, F. Scaling up MIMO: Opportunities and Challenges With Very Large Arrays. IEEE Signal Process. Mag. 2013, 30, 40-60. [CrossRef]

[5] Larsson, E.G.; Tufvesson, F.; Edfors, O.; Marzetta, T.L. Massive MIMO for Next Generation Wireless Systems. IEEE Commun. Mag. 2014, 52, 186-195. [CrossRef]

[6] Marzetta, T.L. Massive MIMO: An Introduction. Bell Labs Tech. J. 2015, 20, 11-22. [CrossRef]

[7] Wu, X.; Beaulieu, N.C.; Liu, D. On Favorable Propagation in Massive MIMO Systems and Different Antenna Configurations. IEEE Access 2017, 5, 5578-5593. [CrossRef]

[8] Karthik Kumar Vaigandla and Dr.N.Venu, "A Survey on Future Generation Wireless Communications - 5G : Multiple Access Techniques, Physical Layer Security, Beamforming Approach", Journal of Information and Computational Science, Volume 11 Issue 9,2021, pp. 449-474

[9] F. Rusek, D. Persson, B. K. Lau, E. G. Larsson, T. L. Marzetta, O. Edfors, and F. Tufvesson, "Scaling up MIMO: Opportunities and challenges with very large arrays," IEEE Signal Process. Mag., vol. 30, no.1, pp. 40-46, Jan. 2013.

[10] D. Gesbert, M. Kountouris, R. W. Heath, Jr., C. B. Chae, and T.Salzer, "From Single user to Multiuser Communications: Shifting the MIMO paradigm," IEEE Signal Processing Magazine, Vol. 24, No. 5, pp. 36-46, Oct., 2007.

[11] T. L.Marzetta, "Noncooperative cellular wireless with unlimited numbers of base station antennas," IEEE Trans. Wireless Commun., vol. 9, no. 11, pp. 3590-3600, Nov. 2010.

[12] E. G. Larsson, F. Tufvesson, O. Edfors, and T. L. Marzetta, "Massive MIMO for next generation wireless systems, " IEEE Commun. Mag., vol. 52, no. 2, pp. 186-195, Feb. 2014.

[13] Larsson, E.; Edfors, O.; Tufvesson, F.; Marzetta, T. Massive MIMO for next generation wireless Systems. IEEE Commun. Mag. 2014, 52, 186-195.

[14] MARZETTA T L. Noncooperative cellular wireless with unlimited numbers of base station antennas[ J]. IEEE Trans-actions on Wireless Communications, 2010 , 9 ( 11 ) : 3590 - 3600 .

[15] Qiu ling. MIMO communication technology of multi-user and multi-cell [M]. Beijing: people's posts and telecommunications press, 2011.

[16] Swapnaja Deshpande, "Introduction to MIMO and Massive MIMO".

[17] H. Q. Ngo, E. G. Larsson, and T. L. Marzetta, "Energy and spectral efficiency of very large multiuser MIMO systems," IEEE Trans. Commun., vol. 61, no. 4, pp. 1436-1449, Apr. 2013.

[18] Nguyen, M. Massive MIMO: A Survey of Benefits and Challenges. ICSES Trans. Comput. Hardw. Electr. Eng. 2018, 4, 1-4.

[19] Hoydis, J.; Hosseini, K.; Brink, S.T.; Debbah, M. Making smart use of excess antennas: Massive MIMO, small cells, and TDD. Bell Labs Tech. J. 2013, 18, 5-21. [CrossRef] 
[20] Popovski, P.; Stefanovic', C` .; Nielsen, J.J.; De Carvalho, E.; Angjelichinoski, M.; Trillingsgaard, K.F.; Bana, A.S. Wireless Access in Ultra-Reliable Low-Latency Communication (URLLC). IEEE Trans. Commun. 2019, 67, 5783-5801. [CrossRef]

[21] Hoydis, J.; Brink, S.T.; Debbah, M. Massive MIMO in the/DL of cellular networks: How many antennas do we need? IEEE J. Sel. Areas Commun. 2013, 31, 160-171. [CrossRef]

[22] Lu Lu, Geoffrey Ye Li, A. Lee Swindlehurst, Alexei Ashikhmin, and Rui Zhang," An Overview of Massive MIMO: Benefits and Challenges", IEEE JOURNAL OF SELECTED TOPICS IN SIGNAL PROCESSING, VOL. 8, NO. 5, 2014. Digital Object Identifier 10.1109/JSTSP.2014.2317671

[23] Moqbel, M.A.M.;Wangdong, W.; Ali, A.Z. MIMO Channel Estimation Using the LS and MMSE Algorithm. IOSR J. Electron. Commun. Eng. 2017, 12, 13-22. [CrossRef]

[24] Robin Chataut and Robert Akl, "Massive MIMO Systems for $5 G$ and beyond NetworksOverview, Recent Trends, Challenges, and Future Research Direction”, Sensors 2020, 20, 2753; doi:10.3390/s20102753

[25] Fatema, N.; Hua, G.; Xiang, Y.; Peng, D.; Natgunanathan, I. Massive MIMO Linear Precoding: A Survey. IEEE Syst. J. 2018, 12, 3920-3931. [CrossRef]

[26] E. Björnson, J. Hoydis, M. Kountouris, and M. Debbah, "Massive MIMO systems with non-ideal hardware: Energy efficiency, estimation, and capacity limits," IEEE Trans. Inf. Theory, 2013.

[27] Studer, C.; Wenk, M.; Burg, A. MIMO transmission with residual transmit-RF impairments. In Proceedings of the ITG/IEEEWorkshop on Smart Antennas (WSA), Bremen, Germany, 23-24 February 2010.

[28] C. Studer and E. G. Larsson, "PAR-aware large-scale multi-user MIMO-OFDM downlink,” IEEE J. Sel. Areas Commun., vol. 31, no. 2, pp. 303-313, Feb. 2013

[29] Ghacham, S.; Benjillali, M.; Guennoun, Z. Low-complexity detection for massive MIMO systems over correlated Rician fading. In Proceedings of the 2017 13th InternationalWireless Communications and Mobile Computing Conference (IWCMC), Valencia, Spain, 26-30 June 2017; pp. 1677-1682.

[30] Torres, P.; Charrua, L.; Gusmao, A. On the Uplink Detection Performance in MUMIMO Broadband Systems with a Large Number of BS Antennas. In Proceedings of the OFDM 2014 18th International OFDM Workshop 2014 (InOWo’14), Essen, Germany, 27-28 August 2014; pp. 1-8.

[31] Gao, X.; Dai, L.; Ma, Y.;Wang, Z. Low-complexity near-optimal signal detection for uplink large-scale MIMO systems. Electr. Lett. 2014, 50,1326-1328. [CrossRef]

[32] Kim, Y.; Seo, J.H.; Kim, H.M.; Kim, S. Soft linear MMSE detection for coded MIMO systems. In Proceedings of the 2013 19th Asia-Pacific Conference on Communications (APCC), Denpasar, Indonesia, 29-31 August 2013; pp. 657-660.

[33] Y.-H. Nam, B. L. Ng, K. Sayana, Y. Li, J. Zhang, Y. Kim, and J. Lee, "Full-dimension MIMO FD-MIMO for next generation cellular technology," IEEE Commun. Mag., vol. 51, no. 6, pp. 172-179, Jun. 2013.

[34] P. S. Taluja and B. L. Hughes, "Diversity limits of compact broadband multi-antenna systems, ” IEEE J. Sel. Areas Commun., vol. 31, no. 2, pp. 326-337, Feb. 2013.

[35] Y. Zeng, R. Zhang, and Z.N. Chen, "Electromagnetic lens-focusing antenna enabled massive MIMO," in Proc. IEEE/CIC Int.Conf.Commun. in China (ICCC), Xi'an, China, Aug. 2013, pp. 454-459.

[36] Y. Zeng, R. Zhang, and Z. N. Chen, "Electromagnetic lens-focusing enabled massive MIMO: Performance improvement and cost reduction," 2013 [Online]. Available: http://arxiv.org/abs/1312.0718. 
[37] Noor Hidayah Muhamad Adnan, Islam Md. Rafiqul and AHM Zahirul Alam, "Massive MIMO for Fifth Generation (5G): Opportunities and Challenges", International Conference on Computer \& Communication Engineering, 2016, DOI 10.1109/ICCCE.2016.23.

[38] Noha Hassan and Xavier Fernando, "Massive MIMO Wireless Networks: An Overview”, Electronics 2017, 6, 63; doi:10.3390/electronics6030063

[39] Karthik Kumar Vaigandla, Mounika Siluveru and Sandhya Rani Bolla, "Analysis of PAPR and Beamforming For 5G MIMO-OFDM", International journal of analytical and experimental modal analysis, Volume XII, Issue X, 2020, pp.483-490.

[40] Allanki Sanyasi Rao, V Karthik Kumar, Malothu Devsingh, "Channel Capacity with and without CSIT for different configurations of MIMO System”, The International journal of analytical and experimental modal analysis, Volume XIII, Issue I, 2021, PP.48-55.

[41] Karthik Kumar Vaigandla, RadhaKrishna Karne, Allanki Sanyasi Rao, "Analysis of MIMO-OFDM: Effect of Mutual Coupling, Frequency Response, SNR and Channel Capacity", YMER Digital, VOLUME 20 : ISSUE 10 ,2021, pp.118-126.

[42] Karthik Kumar Vaigandla, SandyaRani Bolla, RadhaKrishna Karne, "A Survey on Future Generation Wireless Communications-6G: Requirements, Technologies, Challenges and Applications", International Journal of Advanced Trends in Computer Science and Engineering, Volume 10, No.5, September - October 2021, pp.3067-3076. 